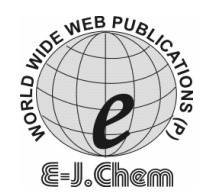

http://www.e-journals.net
ISSN: 0973-4945; CODEN ECJHAO

E-Journal of Chemistry

2009, 6(S1), S147-S152

\title{
Antimony Selenide Crystals Encapsulated within Single Walled Carbon Nanotubes-A DFT Study
}

\author{
NAVARATNARAJAH KUGANATHAN \\ Department of Chemistry, \\ University of Oxford, Oxford, OX1 3QR, UK. \\ Currently at Department of Chemistry, \\ University of Bath, Bath, BA2 7AY, UK \\ nk247@bath.ac.uk
}

Received 27 March 2009; Accepted 20 May 2009

\begin{abstract}
The structure and binding energies of antimony selenide crystals encapsulated within single-walled carbon nanotubes are studied using density functional theory. Calculations were performed on the simulated $\mathrm{Sb}_{2} \mathrm{Se}_{3}$ structure encapsulated within single walled nanotube to investigate the perturbations on the $\mathrm{Sb}_{2} \mathrm{Se}_{3}$ crystal and tube structure and electronic structure and to estimate the binding energy. The calculated structures are in good agreement with the experimental high resolution transmission electron microscopy images of the $\mathrm{Sb}_{2} \mathrm{Se}_{3} @ \mathrm{SWNT}$. The calculated binding energy shows that larger diameter tube could accommodate the $\mathrm{Sb}_{2} \mathrm{Se}_{3}$ crystals exothermically. Minimal charge transfer is observed between nanotube and the $\mathrm{Sb}_{2} \mathrm{Se}_{3}$ crystals.
\end{abstract}

Keywords: DFT, Nanotubes, Antimony, Selenium, Single walled carbon nanotubes.

\section{Introduction}

The synthesis of inorganic nano-crystals encapsulated in single-walled carbon nanotubes has been considered as a possible route for studying the properties and applications of lowdimensional materials. A wide variety of metal halides, metal oxides and other materials have been introduced into open multi walled or single walled carbon nanotubes (SWNTs) from a melt e.g. KI, $\mathrm{HgTe}, \mathrm{TbCl}_{3}$ and $\mathrm{Sb}_{2} \mathrm{O}_{3}{ }^{1-10}$. In many cases the encapsulation of these salts introduces a change of the structure of the included material relative to its bulk form. For example, the encapsulation of KI in SWNTs yields a structure without an overall change but with a systematic reduction of coordination ${ }^{7}$. A detailed structural analysis of HgTe@SWNT showed that coordination of $\mathrm{Hg}$ and Te was altered significantly from the tetrahedral coordination found in the bulk HgTe zinc blende structure to trigonal planar and calculations showed ${ }^{8}$ that $1 \mathrm{D} \mathrm{HgTe}$ crystals are semi conducting with a band gap of ca $1.2 \mathrm{eV}$. 
But in contrast ${ }^{11}$, bulk HgTe is a semi metal with the band gap of $-0.303 \mathrm{eV}$. Theoretical calculations of nano-crystal-SWNT composites clearly demonstrate the unique low dimensional structures and properties of intercalated materials.

Recently, Tsung-wu Lin et al. ${ }^{12}$ have filled nanotubes with $\mathrm{Sb}_{2} \mathrm{Se}_{3}$ and recorded its images using high resolution transmission electron microscopy (HRTEM). Close examination of the encapsulated crystal shows every four dark spots can form a unit with the shape of parallelogram and this unit is regularly repeated along the tube axis. The spots across the SWNT capillary are spaced at average intervals of $0.38 \mathrm{~nm}$, and along the SWNT capillary the spacing increases to $0.4 \mathrm{~nm}$. Figure 1 shows the model structure of $\mathrm{Sb}_{2} \mathrm{Se}_{3} @ \mathrm{SWNT}$ derived from the HRTEM. The diameter of the observed SWNT is reported to be ca $1.23 \mathrm{~nm}$, which is close to the diameter of a $(9,9)$ SWNT.

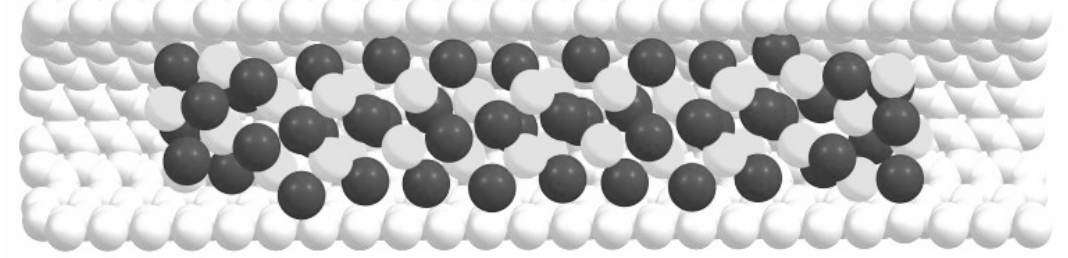

Figure 1. Model structure of $\mathrm{Sb}_{2} \mathrm{Se}_{3}$ crystals encapsulated within single walled carbon nanotube. (Sb-Yellow (Y), Se- Green (G)).

In this paper, we use density functional methods, as embedded in the SIESTA code, to test the proposed model theoretically and investigate the perturbations on the molecular and electronic structure of the crystal and the SWNT and the energy of formation of the $\mathrm{Sb}_{2} \mathrm{Se}_{3} @ \mathrm{SWNT}$ composite. Mulliken population analysis is performed to account the charge transfer between nanotube and the crystals.

\section{Theoretical procedures}

In order to investigate the structural, electronic behaviour of $\mathrm{Sb}_{2} \mathrm{Se}_{3}$ encapsulated within single walled carbon nanotubes, $a b$ initio total energy calculations, based on density functional theory are employed. We have used SIESTA code which performs full selfconsistent calculations solving Kohn-Sham (KS) equations ${ }^{13-15}$. For the exchange correlation term Generalised Gradient Approximation (GGA) is proposed as by Perdew-Burke-Ernzerhof $(\mathrm{PBE})^{16}$. Core electrons are replaced by non local standard norm conserving Troullier-Martins pseudo potentials for $\mathrm{C}, \mathrm{Sb}$ and $\mathrm{Se}^{17-19}$. The reference electronic configuration, cut-off radius and partial core cut-off radius for all pseudo potentials employed here are tabulated Table 1. For $\mathrm{C}, \mathrm{Sb}$ and $\mathrm{Se}$, a partial core correction is necessary to account for nonlinearity of the exchange and correlation potential between core and valence charge densities. Both the $\mathrm{Sb}$ and Se pseudo potentials were generated with scalar relativistic effects.

Table 1. Reference configuration and cut-off radii (a.u.) of the pseudo potentials used in this study.

\begin{tabular}{ccccc}
\hline Atom & $\mathrm{C}$ & $\mathrm{Sb}$ & $\mathrm{Se}$ \\
\hline Reference & & $2 s^{2} 2 p^{2} 3 d^{0} 4 f^{0}$ & $5 s^{2} 5 p^{3} 5 d^{0} 4 f^{0}$ & $4 s^{2} 4 p^{4} 5 d^{0} 4 f^{0}$ \\
Core radius (a.u.) & $s$ & 1.25 & 1.80 & 1.40 \\
& $p$ & 1.25 & 1.90 & 1.60 \\
& $d$ & 1.25 & 3.00 & 2.00 \\
Core cutoff (a.u.) & $f$ & 1.25 & 2.10 & 2.00 \\
& & 1.50 & 3.50 & 1.235 \\
\hline
\end{tabular}


The quality of the pseudo potential was tested comparing the eigen values and excitation energies of all electron calculations on the same series of atomic configurations. The crossexcitation energies for $\mathrm{Sb}$ and $\mathrm{Se}$ pseudo potentials were not more than $0.004 \mathrm{eV}$ and for $0.008 \mathrm{eV}$ respectively and for $\mathrm{C} \leq 0.09 \mathrm{eV}$, indicating the excellent transferability of these pseudo potentials.

The one-electron Kohn-Sham eigen states were expanded in a basis of strictly localized numerical atomic orbitals (NAOs) ${ }^{20}$. For carbon, a double- $\zeta$ basis set for $2 s$ and $2 p$ valence states and a single- $\zeta$ basis set for $3 d$ were used. For $\mathrm{Sb}$, a single- $\zeta$ basis set for the $5 d$ and a double $-\zeta$ basis set for the $5 s$ and $5 p$ orbitals were used. For Se a double- $\zeta$ basis set for the $4 s$ and $4 p$ orbitals and a single- $\zeta$ basis set for the $4 d$ orbitals were used.

Structure optimizations were performed using a conjugate gradient algorithm and the forces on the atoms were obtained from the Hellman-Feynman theorem including Pulay corrections. In all optimised structures forces on the atoms were smaller than $0.18 \mathrm{eV} / \AA$ and the stress tensor was less than 0.05 GPa.

To represent the charge density a cut-off of 200-300 Ry for the real space grid integration was used in all calculations. A single k-point $(\Gamma)$ was used for all calculations on molecular $\mathrm{Sb}_{2} \mathrm{Se}_{3}$ crystals and for the $\mathrm{Sb}_{2} \mathrm{Se}_{3} @ \mathrm{SWNT}$ composites. In calculations on $\mathrm{Sb}_{2} \mathrm{Se}_{3}$ with periodic boundary conditions, reciprocal space was sampled at between 1-5 k- points using the method of Monkhorst and Pack ${ }^{21}$.

For calculations on infinite $\mathrm{Sb}_{2} \mathrm{Se}_{3}$ crystals and $\mathrm{Sb}_{2} \mathrm{Se}_{3} @ \mathrm{SWNT}$ composites, periodic boundary conditions were applied to enforce a minimum lateral separation of $25 \AA$ between structures in adjacent unit cells. At this separation the interaction between these structures and their periodic images are negligible.

\section{Results and Discussion}

$\mathrm{Sb}_{2} \mathrm{Se}_{3}$ is a layer-structured compound and has an orthorhombic lattice ${ }^{22}$. In order to test the quality of the pseudo potentials and the basis sets, structural relaxation was performed on bulk $\mathrm{Sb}_{2} \mathrm{Se}_{3}$ orthorhombic structure. Calculated lattice parameters $(\mathrm{a}=11.91 \AA \mathrm{A}, \mathrm{b}=3.93 \AA$, $\mathrm{c}=11.70 \AA$ and $\alpha, \beta$ and $\left.\gamma=90^{\circ}\right)$ are in good agreement with the experiment $(\mathrm{a}=11.79 \AA$, $\mathrm{b}=3.98 \AA, \mathrm{c}=11.65 \AA$ and $\alpha, \beta$ and $\left.\gamma=90^{\circ}\right)^{23}$ thereby validating our choice of pseudopotentials and basis sets.

As we have discussed in the previous papers, ${ }^{24,25}$ the encapsulated crystal has been treated as a molecule, while the nanotube is treated as an infinite system by imposing periodic boundary conditions.

We selected two sizes of SWNTs to encapsulate the $\mathrm{Sb}_{32} \mathrm{Se}_{48}$ nanocrystals. They are $(9,9)$ and $(17,0)$ with diameters of $12.36 \AA$ and $13.53 \AA$ respectively. The selection of the $(9,9)$ tube is based on the report by Tsung-wu $\operatorname{Lin}^{12}$ who used HRTEM measurements to determine the diameter of the SWNT. The selection of $(17,0)$ is arbitrary and this tube has a wider diameter than the $(9,9)$. The purpose of this selection of a wider diameter tube is to compare the structural distortions and binding nature with the smaller tube. Table 2 shows the composition of the super cells used to model the $\mathrm{Sb}_{32} \mathrm{Se}_{48} @ \mathrm{SWNT}$ composites.

Table 2. Composition of super cells used to model $\mathrm{Sb}_{32} \mathrm{Se}_{48} @ \mathrm{SWNT}$ composites.

\begin{tabular}{ccccc}
\hline $\begin{array}{c}\text { Type of } \\
\text { nanotube }\end{array}$ & $\begin{array}{c}\text { Diameter of } \\
\text { nanotubes }\left(\mathrm{d}_{\mathrm{t}}\right)(\mathrm{A})\end{array}$ & $\begin{array}{c}\text { Number of carbon } \\
\text { atoms in the pristine } \\
\text { nanotube }\end{array}$ & $\begin{array}{c}\text { Number of atoms in Number of atoms in the } \\
\text { the } \mathrm{Sb}_{32} \mathrm{Se}_{48} \text { crystal }\end{array}$ & $\begin{array}{c}\mathrm{Sb}_{32} \mathrm{Se}_{48} @ \mathrm{SWNT} \\
\text { composite }\end{array}$ \\
\hline$(9,9)$ & 12.36 & 864 & 80 & 944 \\
$(17,0)$ & 13.53 & 884 & 80 & 964 \\
\hline
\end{tabular}


The final fully relaxed structures of $\mathrm{Sb}_{32} \mathrm{Se}_{48} @ \mathrm{SWNTs}$ are shown in Figure 2.

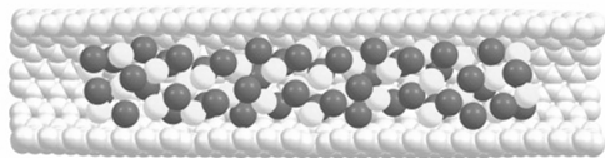

(a)

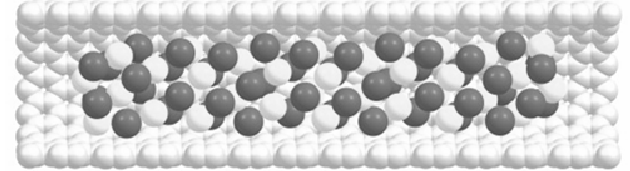

(b)
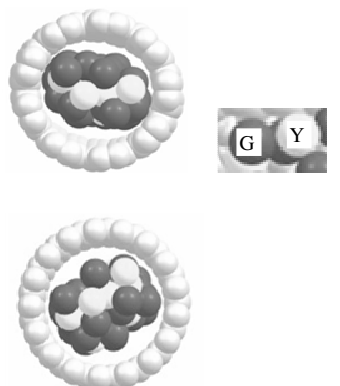

Figure 2. (a). Optimised structures of (a). $\mathrm{Sb}_{32} \mathrm{Se}_{48} @(9,9)$ (b). $\mathrm{Sb}_{32} \mathrm{Se}_{48} @(17,0)$.

Geometrical parameters for the central motifs of the $\mathrm{Sb}_{32} \mathrm{Se}_{48}$ crystals in the structural optimizations of $\mathrm{Sb}_{32} \mathrm{Se}_{48} @ \mathrm{SWNT}$ [SWNT= $\left.(9,9),(17,0)\right]$ and isolated $\mathrm{Sb}_{32} \mathrm{Se}_{48}$ crystal are shown in Table 3 and Table 4.

Table 3. Structural parameters (bond lengths) calculated for $\mathrm{Sb}_{32} \mathrm{Se}_{48} @ \mathrm{SWNT}$. [SWNT $=(9,9)$ and $(17,0)]: d_{t}$ is the diameter of the (empty) SWNT; Structural data for an isolated and model $\mathrm{Sb}_{32} \mathrm{Se}_{48} @ \mathrm{SWNT}$ structure are also provided.

\begin{tabular}{ccc}
\hline Type & $d_{t}(\AA)$ & $\mathrm{Sb}-\mathrm{Se}(\AA)$ \\
\hline $\mathrm{Sb}_{32} \mathrm{Se}_{48} @(9,9)$ & 12.36 & $2.67,2.68,2.70,2.72$ \\
$\mathrm{Sb}_{32} \mathrm{Se}_{48} @(17,0)$ & 13.53 & $2.69,2.70,2.71,2.73$ \\
${\mathrm{Isolated} \mathrm{Sb}_{32} \mathrm{Se}_{48}}_{\text {Isto }}$ & - & $2.69,2.70$ \\
Experiment & - & 2.67 \\
\hline
\end{tabular}

Table 4. Structural parameters (bond angles) calculated for $\mathrm{Sb}_{32} \mathrm{Se}_{48} @ \mathrm{SWNT}$. $[$ SWNT $=(9,9)$ and $(17,0)]$

\begin{tabular}{|c|c|c|c|}
\hline Type & $d_{t}(\AA)$ & Bond angle $(\mathrm{Se}-\mathrm{Sb}-\mathrm{Se})\left({ }^{\circ}\right)$ & Bond angle $(\mathrm{Sb}-\mathrm{Se}-\mathrm{Sb})\left(^{\circ}\right)$ \\
\hline $\mathrm{Sb}_{32} \mathrm{Se}_{48} @(9,9)$ & 12.36 & $87.8,88.4,97.5,98.2$ & $88.3,92.0,92.2,103.1$ \\
\hline $\mathrm{Sb}_{32} \mathrm{Se}_{48} @(17,0)$ & 13.53 & $90.1,92.3,94.6,95.3$ & $91.1,92.9,96.3,101.5$ \\
\hline Isolated $\mathrm{Sb}_{32} \mathrm{Se}_{48}$ & - & $93.5,94.1,96.4,97.3$ & $90.9,91.1,92.8,98.0$ \\
\hline Experiment & - & 88.3 & 98.8 \\
\hline
\end{tabular}

The calculated Sb-Se bond lengths and angles in the composites are in good agreement with the proposed model structure. The calculated deviations in bond lengths and angles are $\pm 0.06 \AA$ and $\pm 10^{\circ}$ respectively. According to the calculated parameters, for the smallest diameter tube $(9,9), \mathrm{Sb}-\mathrm{Se}$ bond lengths are slightly shorter than the isolated $\mathrm{Sb}_{32} \mathrm{Se}_{48}$ structure. But, for the largest tube, $(17,0)$, values are nearly same. Although, Sb-Se bond lengths show some evidence of distortion, more evident are changes in the $\mathrm{Sb}$-Se bond angles. If we compare the $\mathrm{Sb}$-Se bond angles in the composites with the isolated $\mathrm{Sb}_{32} \mathrm{Se}_{48}$ structure, for the smallest tube Se-Sb-Se angles are smaller than the angles found in the isolated structure. But Sb-Se-Sb angle values are nearly the same in both cases. For the largest tube, $(17,0)$, the angle values approach the values found in the isolated structure, indicating that the crystal experiences only small distortions.

Table 5. Sb-C and Se-C distances in the $\mathrm{Sb}_{32} \mathrm{Se}_{48} @ \mathrm{SWNT}$.

\begin{tabular}{ccc}
\hline Composites & Sb-C $(\AA)$ & Se-C $(\AA)$ \\
\hline $\mathrm{Sb}_{32} \mathrm{Se}_{48} @(9,0)$ & $3.17-3.77$ & $3.15-3.51$ \\
$\mathrm{Sb}_{32} \mathrm{Se}_{48} @(17,0)$ & $3.46-3.78$ & $3.20-3.83$ \\
\hline
\end{tabular}


In both cases, $\mathrm{Sb} / \mathrm{Se}-\mathrm{C}$ distances are quite long indicating that there are no chemical bonds. However, Sb-C distances and Se-C distances are shorter in the $(9,9)$ tube than the $(17,0)$ tube. This information indicates that the tube-wall interaction is not large but that distortion is higher in smaller tube. The closest $\mathrm{Sb}-\mathrm{C}$ and $\mathrm{Se}-\mathrm{C}$ distances in the $\mathrm{Sb}_{32} \mathrm{Se}_{48} @ \mathrm{SWNT}$ are given in Table 5 .

The binding energy for $\mathrm{Sb}_{32} \mathrm{Se}_{48}$ nanocrystals encapsulated in SWNTs is given in Table 6. Binding energies were corrected for basis set superposition errors (BSSE) by the counterpoise correction method of Boys and Bernardi ${ }^{26}$ using "ghost" atoms.

Table 6. The binding energy for $\mathrm{Sb}_{32} \mathrm{Se}_{48}$ nanocrystals encapsulated in SWNTs

\begin{tabular}{cccccc}
\hline Type & $E_{b(\text { unc })}$ & $E_{b(C P)}$ & $\Delta E_{S b 2 S e 3 \text { dis }}$ & $\Delta E_{S W N T \text { dis }}$ & $E_{b(\text { corr })}$ \\
\hline $\mathrm{Sb}_{32} \mathrm{Se}_{48} @(9,9)$ & -0.938 & +0.21 & 0.12 & 0.098 & +0.428 \\
$\mathrm{Sb}_{32} \mathrm{Se}_{48} @(17,0)$ & -0.770 & +0.13 & 0.02 & 0.01 & +0.160
\end{tabular}

(Uncorrected binding energy, $E_{b(u n c)}\left(e V / S b_{2} S_{3}\right)$, counterpoise corrected binding energy, $E_{b(C P)}\left(e V / S b_{2} S e_{3}\right)$, distortion energy of $\mathrm{Sb}_{2} \mathrm{Se}_{3}$ crystals, $\Delta E_{S b 2 S e 3 \_d i s}\left(\mathrm{eV} / \mathrm{Sb}_{2} \mathrm{Se}_{3}\right)$ and distortion energy of nanotube, $\Delta E_{S W N T \text { dis }}$ $\left(\mathrm{eV} / \mathrm{Sb}_{2} \mathrm{Se}_{3}\right)$ and the corrected binding energy, $\left.E_{b(\mathrm{corr})}\left(\mathrm{eV} / \mathrm{Sb}_{2} \mathrm{Se}_{3}\right)\right)$.

We find that the BSSE-corrected binding energy is quite endothermic for $\mathrm{Sb}_{32} \mathrm{Se}_{48} @(9,9)$ whereas for $\mathrm{Sb}_{32} \mathrm{Se}_{48} @(17,0)$ the binding energy is approximately zero. When $\mathrm{Sb}_{2} \mathrm{Se}_{3}$ crystals are filled in a smaller diameter tube $[(9,9)]$ more distortion is observed in both crystals and nanotube. The distortion energy of the $(9,9)$ tube is approximately $0.1 \mathrm{eV}$. This higher distortion energy in the nanotube reflects the structure of the nanotube. The tube takes an oval shape to accommodate the $\mathrm{Sb}_{2} \mathrm{Se}_{3}$ crystals. Also the distortion energy of $\mathrm{Sb}_{2} \mathrm{Se}_{3}$ is $0.12 \mathrm{eV}$ for $\mathrm{Sb}_{32} \mathrm{Se}_{48} @(9,9)$. This energy is higher than the energy found in the $(17,0)$ tube. For the larger diameter tube small distortions are observed in both $\mathrm{Sb}_{2} \mathrm{Se}_{3}$ crystals and tube.

Table 7 shows the amount of charge transfer between the $\mathrm{Sb}_{2} \mathrm{Se}_{3}$ crystals and the tubes using Mulliken population charge analysis. Analysis indicates that there is a negligible charge transfer between the $\mathrm{Sb}_{2} \mathrm{Se}_{3}$ crystals and the tubes and charge transfer behaviour is from tube to $\mathrm{Sb}_{2} \mathrm{Se}_{3}$ crystals.

Table 7. Charges calculated for the encapsulation of $\mathrm{Sb}_{2} \mathrm{Se}_{3}$ crystals with two different nanotubes.

\begin{tabular}{cc}
\hline \multicolumn{1}{c}{ Composite } & Charge /C atom \\
\hline $\mathrm{Sb}_{32} \mathrm{Se}_{48} @(9,9)$ & 0.0050 \\
$\mathrm{Sb}_{32} \mathrm{Se}_{48} @(17,0)$ & 0.0040 \\
\hline
\end{tabular}

For both composites, encapsulation of $\mathrm{Sb}_{2} \mathrm{Se}_{3}$ crystals introduces a decrease of electron density in the SWNT. The magnitude of the decrease for the SWNT depends upon the diameter of nanotubes. In a smaller diameter of nanotube a greater degree of charge transfer is observed. In this study, $\mathrm{Sb}_{32} \mathrm{Se}_{48} @(9,9)$ and $\mathrm{Sb}_{32} \mathrm{Se}_{48} @(17,0)$ exhibited $5 \times 10^{-3}$ e per $\mathrm{C}$ atom and $4 \times 10^{-3} e$ per $\mathrm{C}$ atom respectively transferred from the nanotube. Raman spectra of this composite suggested a negligible charge transfer between nanotubes and $\mathrm{Sb}_{2} \mathrm{Se}_{3}$ crystals. This is comparable with our calculation.

\section{Conclusion}

In summary, ab initio calculations, based on the density-functional theory, were used to test the model $\mathrm{Sb}_{2} \mathrm{Se}_{3}$ structure and describe the electronic structure of $\mathrm{Sb}_{2} \mathrm{Se}_{3}$ crystals encapsulated within two different SWNTs. Upon the encapsulation in both nanotubes the binding energy is endothermic. But when tube diameter increases, the binding becomes stronger. For the smaller diameter tube, overall considerable distortions are predicted for both 
the $\mathrm{Sb}_{2} \mathrm{Se}_{3}$ crystals and the nanotube. Less distortion is observed for the larger diameter tube. However structural parameters in the central part of the $\mathrm{Sb}_{32} \mathrm{Se}_{48}$ motif are comparable with the structure proposed from a HRTEM study and they are close to the experimentally reported values for the larger diameter tube. We anticipate that larger diameter tubes can accommodate the $\mathrm{Sb}_{32} \mathrm{Se}_{48}$ crystals exothermically with more accurate spot separations. Minimal charge transfer is observed between the nanotube and the $\mathrm{Sb}_{32} \mathrm{Se}_{48}$ crystal.

\section{Acknowledgement}

The author thanks Tsung-Wu for providing information about the structure of $\mathrm{Sb}_{2} \mathrm{Se}_{3} @ \mathrm{SWNT}$ and Felix trust for funding.

\section{References}

1 Sloan J, Wright DM, Bailey S, Brown G, York A P E, Coleman K S, Green M L H, Hutchison J and Woo H-G, Chem Commun., 1999, 699.

2 Meyer R R, Sloan J, Dunin-Borkowski R E, Kirkland A I, Novotny C, Bailey S R, Hutchison J L and Green M L H, Science, 2000, 1324, 2000.

3 Flahaut E, Sloan J, Friedrichs S and Kirkland A I, Coleman K S, Williams VC, Hanson N, Hutchison J L and Green M L H, Chem Mater., 2006, 18, 2059.

4 Sloan J, Grosvenor S J, Friedrichs S, Kirkland A I, Hutchison J L and Green M. L H, Angew Chem Int Ed., 2002, 41, 1156.

5 Sloan J, Friedrichs S, Meyer R R, Kirkland A I, Hutchison J L and Green M L H, Inorg Chim Acta, 2002, 330, 1.

6 Sloan J, Carter R, Meyer R R, Vlandas A, Kirkland A I, Lindan P J D, Lin G, Harding J and Hutchison J L, Phys Status Solidi B: Basic Solid State Physics, 2006, 243, 3257.

7 Sloan J, Kirkland A I, Hutchison J L and Green M L H, Chem Commun., 2002, 1319.

8 Carter R, Sloan J, Kirkland A I, Meyer R R, Lindan P J D, Lin G, Green M L H and Vlandas A, Hutchison J L and Harding J, Phys Rev Lett., 2006, 96, 215501.

9 Sloan J, Kirkland A I, Hutchison J L and Green M L H, Acc Chem Res., 2002, 35, 1054

10 Sloan J, Luzzi D E, Kirkland A I, Hutchison J L and Green M L H, Mat Res Bull., 2004, 29, 265.

11 Schulman J N and Chang Y C, Phys Rev B., 1986, 33, 2594.

12 TSung-Wu Lin., D Phil., Thesis, University of Oxford, 2007.

13 Artacho E, Sanchez-Portal D, Ordejon P, Garcia A and Soler J M, Phys Status Solidi B: Basic Research., 1999, 215, 809.

14 Ordejon P, Sanchez-Portal D, Garcia A, Artacho E, Junquera J and Soler J M, RIKEN Review., 2000, 29, 42.

15 Soler J M, Artacho E, Gale J D, Garcia A, Junquera J, Ordejon P and Sanchez-Portal D, J Phys: Condens Matter., 2002, 14, 2745.

16 Perdew J P, Burke K and Ernzerhof M, Phys Rev Lett., 1996, 77, 3865.

17 Bachelet G B and Schlueter M, Phys Rev B., 1982, 25, 2103.

18 Fuchs M and Scheffler M, Comput Phys Commun., 1999, 119, 67.

19 Troullier N and Martins J L, Phys Rev B, 1991, 43, 1993.

20 Junquera J, Paz O, Sanchez-Portal D and Artacho E, Phys Rev B, 2001, 64, 235111.

21 Monkhorst H J and Pack J D, Phys Rev B, 1976, 13, 5188.

22 Tideswell N W, Kruse F H and McCullough J D, Acta Cryst., 1957, 10, 99.

23 Caracas R and Gonze X, Phys Chem Minerals., 2005, 32, 295.

24 Kuganathan N and Green J C, Int J Quantum Chem., 2008, 108, 797.

25 Kuganathan N and Green J C, Int J Quantum Chem., 2009, 109, 171.

26 Boys S F and Bernardi F, Mol Phys., 1970, 19, 553. 


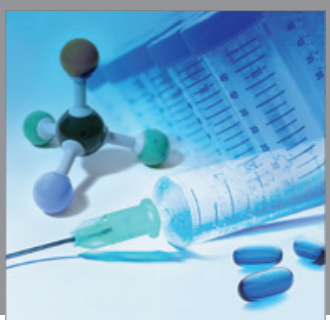

International Journal of

Medicinal Chemistry

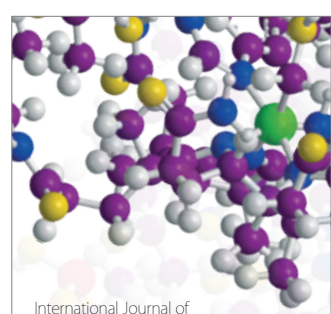

Carbohydrate Chemistry

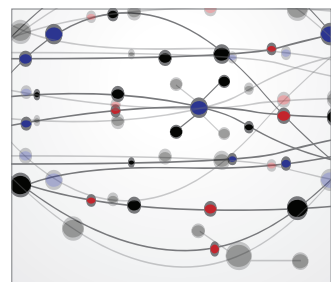

The Scientific World Journal
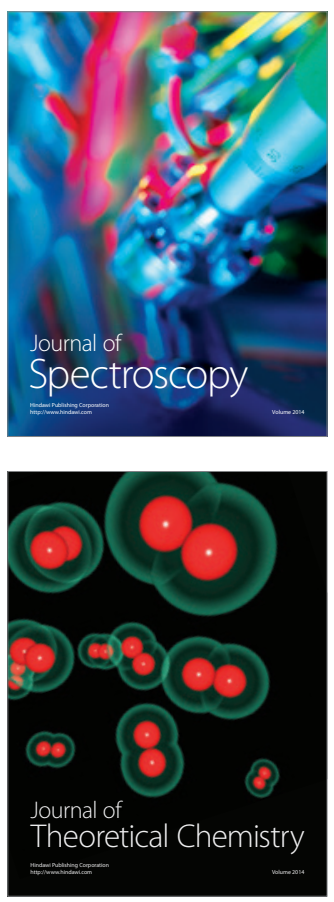
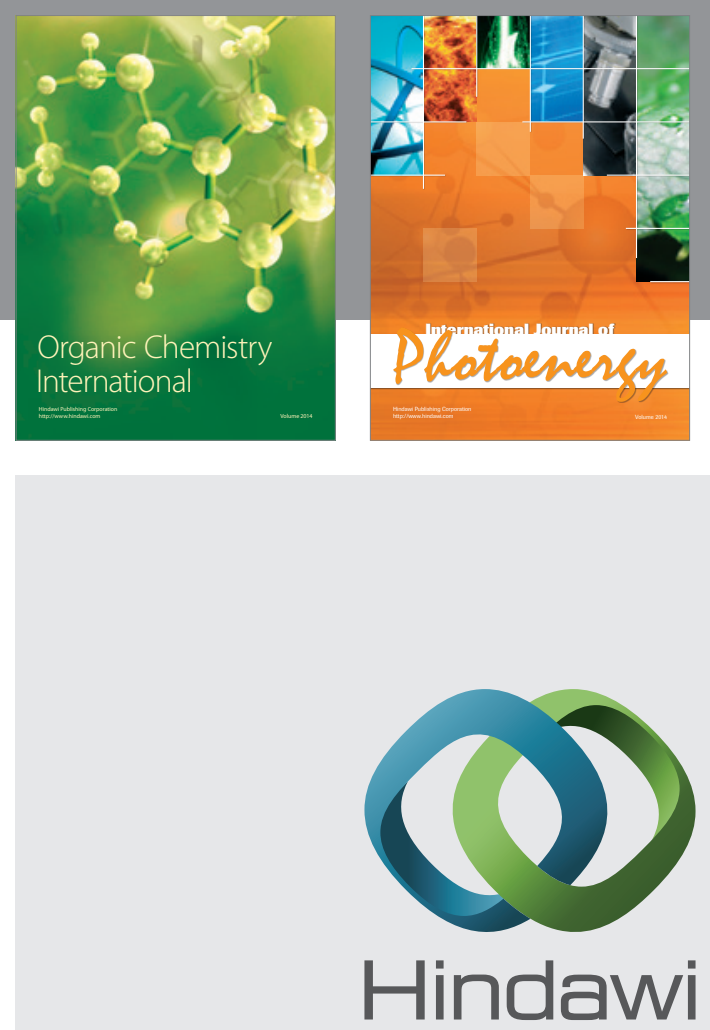

Submit your manuscripts at

http://www.hindawi.com
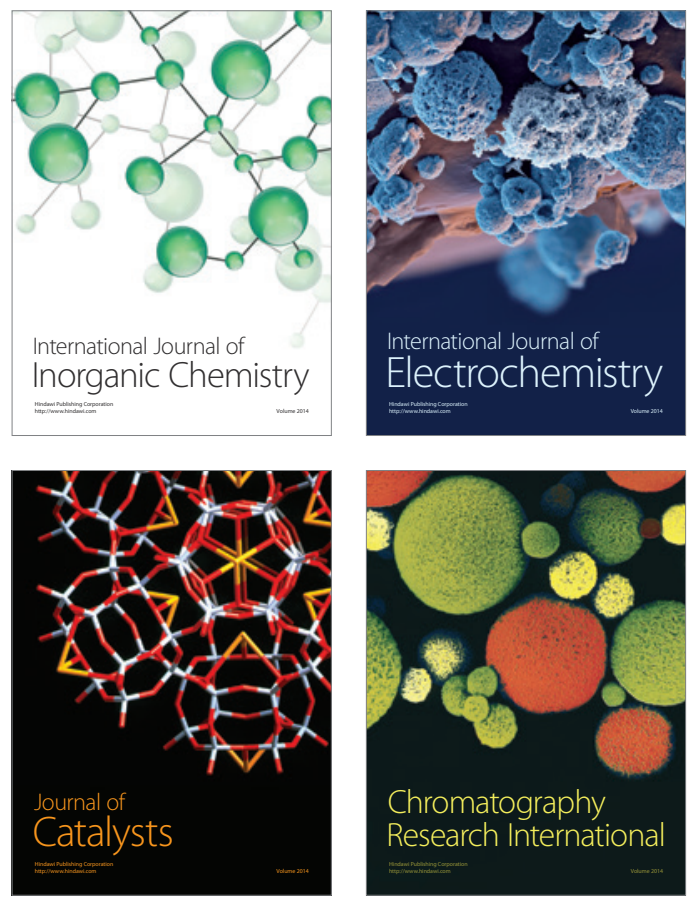
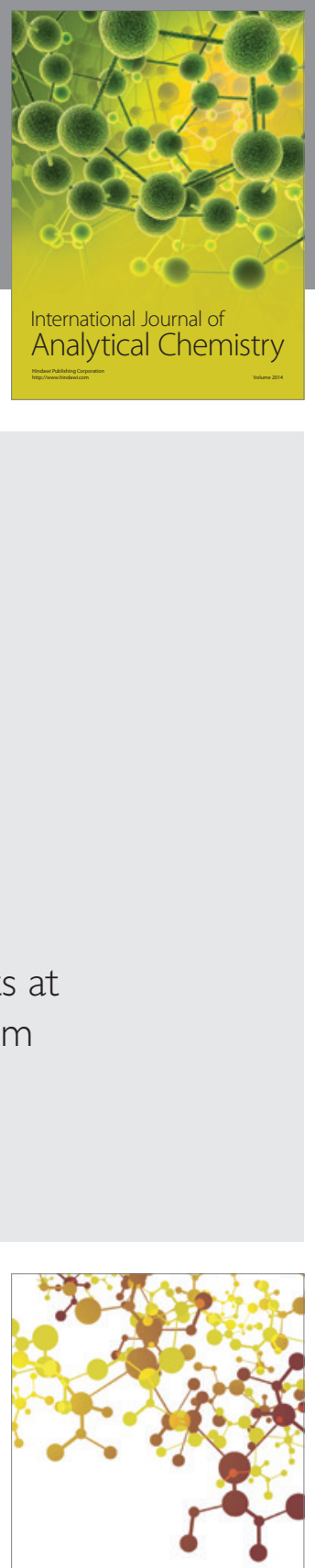

Journal of

Applied Chemistry
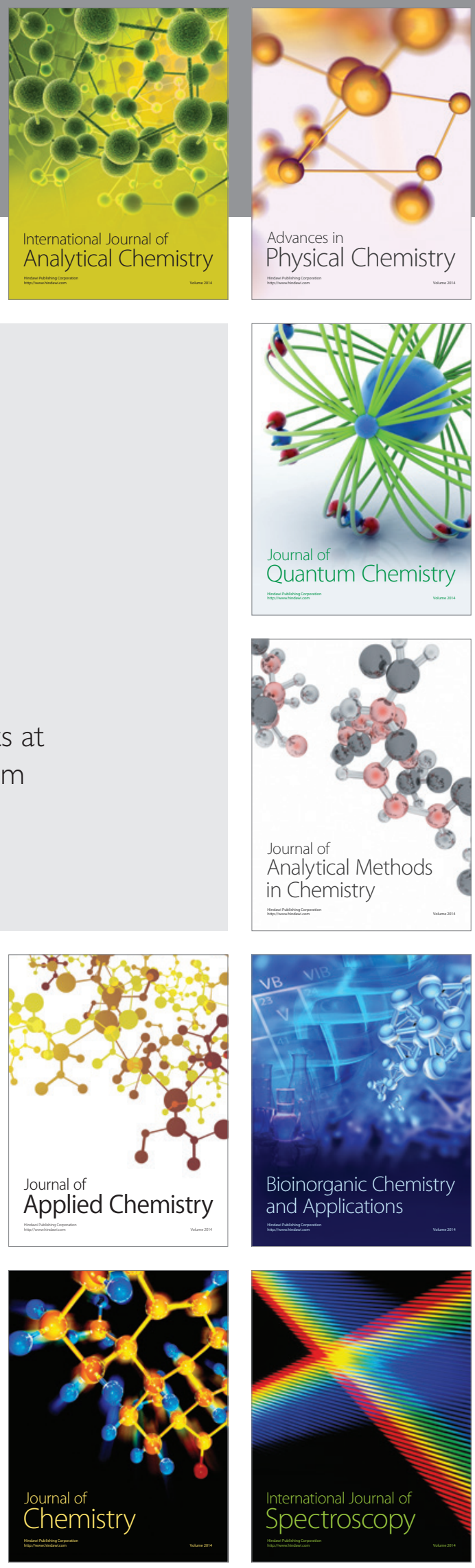